\title{
Free fatty acid binding pocket in the locked structure of SARS-CoV-2 spike protein
}

\author{
Christine Toelzer, ${ }^{1,2 *}$, Kapil Gupta ${ }^{1,2 *}$, Sathish K. N. Yadav ${ }^{1,2 *}$, Ufuk Borucu $^{1,2 *}$, Andrew D. Davidson ${ }^{3}$, Maia $^{2}$ \\ Kavanagh Williamson ${ }^{3}$, Deborah K. Shoemark ${ }^{1,2}$, Frederic Garzoni ${ }^{4}$, Oskar Staufer ${ }^{5,6,7,8}$, Rachel Milligan ${ }^{3}$, Julien \\ Capin $^{1,2}$, Adrian J. Mulholland ${ }^{9}$, Joachim Spatz ${ }^{5,6,7,8}$, Daniel Fitzgerald ${ }^{10}$, Imre Berger , $^{1,2,9}+$, Christiane \\ Schaffitzel ${ }^{1,2} \uparrow$
}

\begin{abstract}
${ }^{1}$ School of Biochemistry, University of Bristol, 1 Tankard's Close, Bristol BS8 1TD, UK. 'Bristol Synthetic Biology Centre BrisSynBio, 24 Tyndall Ave, Bristol BS8 1TQ, UK. ${ }^{3}$ School of Cellular and Molecular Medicine, University of Bristol, University Walk, Bristol, BS8 1TD, UK. ${ }^{4}$ mophoron Ltd, St. Philips Central, Albert Rd, St. Philips, Bristol, BS2 OXJ, UK. ${ }^{5}$ Department for Cellular Biophysics, Max Planck Institute for Medical Research, Jahnstraße 29, 69120 Heidelberg, Germany. ${ }^{6}$ nstitute for Physical Chemistry, Department for Biophysical Chemistry, University of Heidelberg, Im Neuenheimer Feld 253, 69120 Heidelberg, Germany. ${ }^{7}$ Max Planck School Matter to Life, Jahnstraße 29, D-69120 Heidelberg, Germany. ${ }^{8}$ Max Planck Bristol Centre for Minimal Biology, Cantock's Close, Bristol BS8 1TS, UK. ' ${ }^{2}$ chool of Chemistry, University of Bristol, Cantock's Close, Bristol BS8 1TS, UK. ${ }^{10}$ Geneva Biotech Sàrl, Avenue de la Roseraie 64, 1205, Geneva, Switzerland.
\end{abstract}

*These authors contributed equally to this work.

†Corresponding author. Email: cb14941@bristol.ac.uk (C.S.); imre.berger@bristol.ac.uk (I.B.)

COVID-19, caused by severe acute respiratory syndrome-coronavirus-2 (SARS-CoV-2), represents a global crisis. Key to SARS-CoV-2 therapeutic development is unraveling the mechanisms driving high infectivity, broad tissue tropism and severe pathology. Our $2.85 \AA$ cryo-EM structure of SARS-CoV-2 spike (S) glycoprotein reveals that the receptor binding domains (RBDs) tightly bind the essential free fatty acid (FFA) linoleic acid (LA) in three composite binding pockets. The pocket also appears to be present in the highly pathogenic coronaviruses SARS-CoV and MERS-CoV. LA binding stabilizes a locked S conformation giving rise to reduced ACE2 interaction in vitro. In human cells, LA supplementation synergizes with the COVID-19 drug remdesivir, suppressing SARS-CoV-2 replication. Our structure directly links LA and S, setting the stage for intervention strategies targeting LA binding by SARS-CoV-2.

Seven coronaviruses are known to infect humans. The four endemic human coronaviruses OC43, 229E, HKU1 and NL63 cause mild upper respiratory tract infections while pandemic virus SARS-CoV-2, and earlier SARS-CoV and MERS-CoV, can cause severe pneumonia with acute respiratory distress syndrome, multi-organ failure, and death $(1,2)$.

SARS-CoV-2 has acquired functions that promote its harsh disease phenotype. SARS-CoV-2 causes severe inflammation and damage to endothelial cells in the heart, kidneys, liver and intestines, suggestive of a vascular infection rather than a purely respiratory disease $(3,4)$. The attachment of SARS-CoV-2 to a host cell is initiated by the spike protein trimer (S), which decorates the outer surface of the virus, binding to its cognate receptor angiotensin-converting enzyme 2 (ACE2), with higher affinity than SARS-CoV (5-7). A S1/S2 polybasic furin protease cleavage site distinguishes SARSCoV-2 from SARS-CoV or other closely related bat coronaviruses and serves to stimulate entry into host cells and cellcell fusion $(5,8,9)$. Inside the host cell, human coronaviruses remodel the lipid metabolism to facilitate virus replication (10). Infection by SARS-CoV-2 triggers an unusually impaired and dysregulated immune response (11) and a heightened inflammatory response (12) working in synergy with interferon production in the vicinity of infected cells to drive a feed-forward loop to up-regulate ACE2 and further escalate infection (13).

In the search for additional functions that contribute to the pathology of infection, we determined the structure of the SARS-CoV-2 S glycoprotein by cryo-EM (Fig. 1). We produced SARS-CoV-2 S as a secreted trimer (14) in MultiBac (15) baculovirus-infected Hi5 insect cells (fig. S1) (16). Highly purified protein was used for cryo-EM data collection (fig. S2 and table S1). After 3D classification and refinement without applying symmetry (C1) we obtained a $3.0 \AA$ closed conformation from 136,405 particles and a $3.5 \AA$ open conformation with one receptor-binding domain (RBD) in the up position from 57,990 particles (figs. S2 and S3). C3 symmetry was applied to the closed conformation particle pool yielding a $2.85 \AA$ map (Fig. 1A and figs. S2 and S3).

The structure of $\mathrm{S}$ displays the characteristic overall shape observed for coronavirus $\mathrm{S}$ proteins in the closed and open conformations (17-19) with the closed form ( $70 \%)$ predominating in our data set (Fig. 1A and figs. S2 to S4). Model building of the closed form revealed additional density in the RBDs in our structure (Fig. 1B). The tube-like shape of this density was consistent with a fatty acid, with size and shape similar to LA bound to other proteins (Fig. 1B and fig. S5) $(20,21)$. Liquid chromatography coupled ESI-TOF mass spectrometry 
(LS-MS) analysis confirmed the presence of a compound with the molecular weight of LA in our purified sample (Fig. 1C).

Hallmarks of FFA-binding pockets in proteins are an extended "greasy" tube lined by hydrophobic amino acids which accommodates the hydrocarbon tail, and a hydrophilic, often positively charged anchor for the acidic headgroup of the FFA. In our structure, a hydrophobic pocket mostly shaped by phenylalanines forms a bent tube into which the LA fits well (Fig. 1, D and E). The anchor for the headgroup carboxyl is provided by an arginine (R408) and a glutamine (Q409) from the adjacent RBD in the trimer, giving rise to a composite LA-binding site (Fig. 1E). We confirmed the presence of LA in all three binding pockets in the $\mathrm{S}$ trimer in the unsymmetrized (C1) closed structure (fig. S6). Similarly, masked 3D classification focusing on the RBD domains could not identify any unoccupied pockets (fig. S7).

Our S construct contains alterations as compared to native SARS-CoV-2 S namely addition of a trimerization domain and deletion of the polybasic cleavage site, neither of which alter S conformation appreciably $(14,17)$ (fig. S8). Glycosylation sites are located away from the LA-binding pocket and largely native in our structure $(7,17)$ (table S2). Thus, neither mutations nor glycosylation are likely to impact the LAbinding pocket. We compared S and RBD produced in insect cells with mammalian produced $S$ to identify any potential influence of differences in glycosylation on ACE2 binding by competition enzyme-linked immunosorbent assay (ELISA) (Fig. 2A). All three reagents bound ACE2 efficiently. We further confirmed ACE2 binding by S using SEC with purified proteins (Fig. 2B). The LA-binding pocket and the receptor binding motif (RBM) are distal and non-overlapping (Fig. $2 \mathrm{C})$. Notably, in the LA-bound S the RBM is ordered and buried at the interface between RBDs while it was disordered in previously described SARS-CoV-2 S cryo-EM structures $(7,17)$.

SARS-CoV-2 S can also adopt an open conformation (fig. S4) which is compatible with binding ACE2. In previous apo $\mathrm{S}$ cryo-EM structures about $60-75 \%$ of the $\mathrm{S}$ trimers were in the open conformation $(7,17)$, contrasting our observation of $\sim 70 \%$ in the closed conformation. This could be due to LA stabilizing the closed conformation, and if so LA would be expected to reduce ACE2 binding. We performed surface plasmon resonance (SPR) experiments with biotinylated ACE2 immobilized on a streptavidin-coated chip (Fig. 2D and fig. S9). We first determined the $\mathrm{K}_{\mathrm{D}}$ of the RBD/ACE2 interaction to validate our assay. Our value ( $26 \mathrm{nM}$, fig. S9C) is in good agreement with previous studies (44 nM (22)) obtained by SPR with the RBD immobilized and ACE2 as analyte. Apo S was prepared by applying Lipidex, the established method for removing lipids from lipid-binding proteins (23). A $\mathrm{K}_{\mathrm{D}}$ of 0.7 $\mathrm{nM}$ was obtained for the apo S/ACE2 interaction (fig. S9A). For the LA-bound S/ACE2 interaction we obtained a $\mathrm{K}_{\mathrm{D}}$ of 1.4 nM (fig. S9B). We consistently obtained a markedly reduced resonance unit (RU) signal for LA-bound $\mathrm{S}$ as compared to apo $\mathrm{S}$ at the same concentrations (Fig. 2D and fig. S9, A and B). This correlates with the apo state having a higher percentage of S trimers in the open, ACE2-accessible conformation.

We characterized the affinity of the LA interaction both experimentally and computationally. Our SPR assays utilizing immobilized RBD yielded a binding constant of $\sim 41 \mathrm{nM}$ exhibiting a slow off-rate, consistent with tight binding of LA (fig. S10). Repeated molecular dynamics simulations of the entire locked LA-bound spike trimer $(3 \times 100 \mathrm{~ns})$ using GROMACS-2019 (24) corroborated the persistence of stable interactions between LA and the spike trimer (movies S1 and S2). The affinity of LA binding to the spike trimer will likely be higher than to the RBD alone, taking into account polar headgroup interactions with R408 and Q409 of the adjacent RBD (Fig. 1E). The resolution of the RBDs in our open S cryoEM structure was insufficient to either assign, or rule out, a ligand-bound pocket (fig. S3). However, the slow off-rate observed with the RBD monomer (fig. S10) suggests that LA binding could be maintained when the $\mathrm{S}$ trimer transiently converts into the open conformation. This is supported by our observation that LA was retained during $S$ purification in spite of S trimers adopting the open form $\sim 30 \%$ of the time (fig. S2) and by our MD simulations with a modeled ligandbound open spike trimer (movie S3) in which all three LAs remained bound over $500 \mathrm{~ns}$.

Next, we investigated the effect of LA in experiments using live SARS-CoV-2 virus to infect human epithelial cells. Remdesivir is an RNA-dependent RNA polymerase inhibitor and the first anti-viral drug showing benefit in the treatment of COVID-19 in clinical trials, albeit with considerable side effects at the doses required (25). LA supplementation at 50-100 $\mu \mathrm{M}$ concentrations was previously shown to affect coronavirus entry and replication (10). We administered remdesivir at 20,64 and $200 \mathrm{nM}$ concentration, supplementing with $50 \mu \mathrm{M}$ LA (Fig. 2E). Our results revealed synergy, with the dose of remdesivir required to suppress SARS-CoV2 replication markedly reduced by adding LA (Fig. 2, E and F).

We superimposed our LA-bound structure on previous SARS-CoV-2 apo S structures in the closed conformation (7, 17) and identified a gating helix located directly at the entrance of the binding pocket (Fig. 3, A to C). This gating helix, comprising Ty365 and Tyr369, is displaced by about $6 \AA$ when LA is bound, thus opening the pocket (Fig. 3 , A and B). In the apo SARS-CoV-2 $\mathrm{S}$ trimer $(7,17)$, a gap between adjacent RBDs places the hydrophilic anchor residues $\sim 10 \AA$ away from the position of the LA headgroup (Fig. 3C). Upon LA binding, the adjacent RBD in the trimer moves toward its neighbor, and the anchor residues Arg408 and Gln409 lock down on the headgroup of LA (Fig. 3, C and D). Overall, this results in a compaction of trimer architecture in the region 
formed by the three RBDs giving rise to a locked S structure (Fig. 3D and movie S4).

We investigated whether the LA-binding pocket is conserved in the seven coronaviruses that infect humans (Fig. 4A and table S3). Sequence alignment shows that all residues lining the hydrophobic pocket and the anchor residues (Arg408/Gln409) in SARS-CoV-2 are fully conserved in SARSCoV (Fig. 4A). Structural alignment of LA-bound RBDs within the trimer of SARS-CoV-2 and "apo" SARS-CoV RBDs (19) reveals that the LA-binding pocket is present in SARS-CoV. The greasy tube is flanked by a gating helix as in SARS-CoV-2, with Arg395/Gln396 of SARS-CoV positioned $10 \AA$ and $11 \AA$ from the entrance, respectively, virtually identical to apo SARS-CoV-2 (Figs. 3C and 4B). In MERS-CoV, the gating helix and hydrophobic residues lining the pocket are also present. Tyr365, Tyr369 and Phe374 are substituted by likewise hydrophobic leucines and a valine, respectively (Fig. 4, A and C) (19). The Arg408/Gln409 pair is not conserved, however, we identify Asn501/Lys502 and Gln466 as potential anchor residues, located on a $\beta$-sheet and an $\alpha$-helix within the adjacent RBD, up to $11 \AA$ away from the entrance (Fig. 4C). Thus, the greasy tube and hydrophilic anchor appear to be present in MERS-CoV, suggesting convergent evolution. In $\mathrm{HCoV}$ OC43, gating helix and hydrophobic residues lining the pocket are largely conserved, while Tyr365, Tyr369 and Phe374 are replaced by methionines and alanine, respectively (Fig. 4A) (18). Arg413 is located on the same helix as Arg408/Gln409 in SARS-CoV-2 and could serve as a hydrophilic anchor (Fig. 4D). No gap exists in this presumed "apo" form structure between the RBDs which appear already in the locked conformation (Fig. 4D and fig. S11) (18). In HCoV HKU1, the hydrophobic residues are again largely conserved, but a charged residue (Glu375) is positioned directly in front of the entrance, obstructing access for a putative ligand (Fig. 4E) (26). The RBDs of HCoVs 229E and NL63 adopt a very different fold (fig. S13) $(27,28)$, and many of the LA-binding residues are not present (Fig. 4A), hampering predictions of a binding site for fatty acids.

In summary, we find four molecular features mediating LA binding to SARS-CoV-2, and potentially also SARS-CoV and MERS-CoV S proteins: a conserved hydrophobic pocket, a gating helix, amino acid residues pre-positioned to interact with the LA carboxy headgroup, and loosely packed RBDs in the "apo" form. In contrast, in each of the four common circulating $\mathrm{HCoVs}$, it appears that one or more of these four architectural prerequisites are lacking in the $\mathrm{S}$ protein structures (Fig. 4 and figs. S11 and S12). LA binding to SARSCoV-2 $\mathrm{S}$ triggers a locking down of the hydrophilic anchor and a compaction of the RBD trimer (Fig. 3, C and D). In addition to stabilizing the closed conformation, this could also help stabilize the S1 region comprising the N-terminal domain and the RBD. The RBM, central to ACE2 binding, appears to be conformationally preorganized in our structure (Fig. 2C) indicating a generally more rigid RBD trimer when LA is bound. While direct crosstalk in between the LAbinding pocket and the RBM is not apparent from our structure (Fig. 2C), the conformational changes in the RBD trimer (Fig. 3) could impact ACE2 docking and infectivity as indicated by our SPR assays showing reduced levels of S binding in the presence of LA (Fig. 2D). The S protein's tight binding of LA originates from a well-defined size and shape complementarity afforded by the pocket (Fig. 1, B and D). The LAbinding pocket thus presents a promising target for future development of small molecule inhibitors that, for example, could irreversibly lock $\mathrm{S}$ in the closed conformation and interfere with receptor interactions. It is noteworthy in this context that a fatty acid binding pocket was exploited previously to develop potent small molecule anti-viral drugs against rhinovirus, locking viral surface proteins in a conformation incompatible with receptor binding $(29,30)$. These anti-virals were successful in human clinical trials $(31,32)$.

A recent proteomic and metabolomic study of COVID-19 patient sera showed continuous decrease of FFAs including LA (33). Lipid metabolome remodeling is a common element of viral infection $(34,35)$. For coronaviruses, the LA to arachidonic acid metabolic pathway was identified as central to lipid remodeling (10). We hypothesize that LA sequestration by SARS-CoV-2 could confer a tissue-independent mechanism by which pathogenic coronavirus infection may drive immune dysregulation and inflammation (35-37). Our findings provide a direct structural link between LA, COVID-19 pathology and the virus itself and suggest that both the LAbinding pocket within the S protein and the multi-nodal LA signaling axis, represent excellent therapeutic intervention points against SARS-CoV-2 infections.

\section{REFERENCES AND NOTES}

1. P. Zhou, X.-L. Yang, X.-G. Wang, B. Hu, L. Zhang, W. Zhang, H.-R. Si, Y. Zhu, B. Li, C.L. Huang, H.-D. Chen, J. Chen, Y. Luo, H. Guo, R.-D. Jiang, M.-Q. Liu, Y. Chen, X.-R. Shen, X. Wang, X.-S. Zheng, K. Zhao, Q.-J. Chen, F. Deng, L.-L. Liu, B. Yan, F.-X. Zhan, Y.-Y. Wang, G.-F. Xiao, Z.-L. Shi, A pneumonia outbreak associated with a new coronavirus of probable bat origin. Nature 579, 270-273 (2020). doi:10.1038/s41586-020-2012-7 Medline

2. A. Zumla, J. F. Chan, E. I. Azhar, D. S. Hui, K. Y. Yuen, Coronaviruses - drug discovery and therapeutic options. Nat. Rev. Drug Discov. 15, 327-347 (2016) doi:10.1038/nrd.2015.37 Medline

3. Z. Varga, A. J. Flammer, P. Steiger, M. Haberecker, R. Andermatt, A. S. Zinkernagel, M. R. Mehra, R. A. Schuepbach, F. Ruschitzka, H. Moch, Endothelial cell infection and endotheliitis in COVID-19. Lancet 395, 1417-1418 (2020). doi:10.1016/S0140-6736(20)30937-5 Medline

4. V. G. Puelles, M. Lütgehetmann, M. T. Lindenmeyer, J. P. Sperhake, M. N. Wong, L. Allweiss, S. Chilla, A. Heinemann, N. Wanner, S. Liu, F. Braun, S. Lu, S. Pfefferle, A. S. Schröder, C. Edler, O. Gross, M. Glatzel, D. Wichmann, T. Wiech, S. Kluge, K. Pueschel, M. Aepfelbacher, T. B. Huber, Multiorgan and Renal Tropism of SARSCoV-2. N. Engl. J. Med. 383, 590-592 (2020). doi:10.1056/NEJMc2011400 Medline

5. M. Hoffmann, H. Kleine-Weber, S. Schroeder, N. Krüger, T. Herrler, S. Erichsen, T. S. Schiergens, G. Herrler, N.-H. Wu, A. Nitsche, M. A. Müller, C. Drosten, S. Pöhlmann, SARS-CoV-2 Cell Entry Depends on ACE2 and TMPRSS2 and Is 
Blocked by a Clinically Proven Protease Inhibitor. Cell 181, 271-280.e8 (2020). doi:10.1016/i.cell.2020.02.052 Medline

6. M. Letko, A. Marzi, V. Munster, Functional assessment of cell entry and receptor usage for SARS-CoV-2 and other lineage B betacoronaviruses. Nat. Microbiol. 5 , 562-569 (2020). doi:10.1038/s41564-020-0688-y Medline

7. A. C. Walls, Y.-J. Park, M. A. Tortorici, A. Wall, A. T. McGuire, D. Veesler, Structure, Function, and Antigenicity of the SARS-CoV-2 Spike Glycoprotein. Cell 181, 281292.e6 (2020). doi:10.1016/i.cell.2020.02.058 Medline

8. M. Hoffmann, H. Kleine-Weber, S. PöhImann, A Multibasic Cleavage Site in the Spike Protein of SARS-CoV-2 Is Essential for Infection of Human Lung Cells. Mol. Cell 78 779-784.e5 (2020). doi:10.1016/i.molcel.2020.04.022 Medline

9. S. Matsuyama, N. Nao, K. Shirato, M. Kawase, S. Saito, I. Takayama, N. Nagata, T. Sekizuka, H. Katoh, F. Kato, M. Sakata, M. Tahara, S. Kutsuna, N. Ohmagari, M. Kuroda, T. Suzuki, T. Kageyama, M. Takeda, Enhanced isolation of SARS-CoV-2 by TMPRSS2-expressing cells. Proc. Natl. Acad. Sci. U.S.A. 117, 7001-7003 (2020). doi:10.1073/pnas.2002589117 Medline

10. B. Yan, H. Chu, D. Yang, K.-H. Sze, P.-M. Lai, S. Yuan, H. Shuai, Y. Wang, R. Y.-T. Kao, J. F.-W. Chan, K.-Y. Yuen, Characterization of the Lipidomic Profile of Human Coronavirus-Infected Cells: Implications for Lipid Metabolism Remodeling upon Coronavirus Replication. Viruses 11, 73 (2019). doi:10.3390/v11010073 Medline

11. C. Qin, L. Zhou, Z. Hu, S. Zhang, S. Yang, Y. Tao, C. Xie, K. Ma, K. Shang, W. Wang, D.-S. Tian, Dysregulation of immune response in patients with coronavirus 2019 (COVID-19) in Wuhan, China. Clin. Infect. Dis. 71, 762-768 (2020). doi:10.1093/cid/ciaa248 Medline

12. M. Z. Tay, C. M. Poh, L. Rénia, P. A. MacAry, L. F. P. Ng, The trinity of COVID-19: Immunity, inflammation and intervention. Nat. Rev. Immunol. 20, 363-374 (2020). doi:10.1038/s41577-020-0311-8 Medline

13. C. G. K. Ziegler, S. J. Allon, S. K. Nyquist, I. M. Mbano, V. N. Miao, C. N. Tzouanas, Y. Cao, A. S. Yousif, J. Bals, B. M. Hauser, J. Feldman, C. Muus, M. H. Wadsworth 2nd, S. W. Kazer, T. K. Hughes, B. Doran, G. J. Gatter, M. Vukovic, F. Taliaferro, B. E. Mead, Z. Guo, J. P. Wang, D. Gras, M. Plaisant, M. Ansari, I. Angelidis, H. Adler, J. M. S. Sucre, C. J. Taylor, B. Lin, A. Waghray, V. Mitsialis, D. F. Dwyer, K. M. Buchheit, J. A. Boyce, N. A. Barrett, T. M. Laidlaw, S. L. Carroll, L. Colonna, V. Tkachev, C. W. Peterson, A. Yu, H. B. Zheng, H. P. Gideon, C. G. Winchell, P. L. Lin, C. D. Bingle, S. B. Snapper, J. A. Kropski, F. J. Theis, H. B. Schiller, L.-E. Zaragosi, P. Barbry, A. Leslie, H.-P. Kiem, J. L. Flynn, S. M. Fortune, B. Berger, R. W. Finberg, L. S. Kean, M. Garber, A. G. Schmidt, D. Lingwood, A. K. Shalek, J. OrdovasMontanes; HCA Lung Biological Network, SARS-CoV-2 Receptor ACE2 Is an Interferon-Stimulated Gene in Human Airway Epithelial Cells and Is Detected in Specific Cell Subsets across Tissues. Cell 181, 1016-1035.e19 (2020). doi:10.1016/i.cell.2020.04.035 Medline

14. F. Amanat, D. Stadlbauer, S. Strohmeier, T. H. O. Nguyen, V. Chromikova, M. McMahon, K. Jiang, G. A. Arunkumar, D. Jurczyszak, J. Polanco, M. BermudezGonzalez, G. Kleiner, T. Aydillo, L. Miorin, D. S. Fierer, L. A. Lugo, E. M. Kojic, J. Stoever, S. T. H. Liu, C. Cunningham-Rundles, P. L. Felgner, T. Moran, A. GarcíaSastre, D. Caplivski, A. C. Cheng, K. Kedzierska, O. Vapalahti, J. M. Hepojoki, V. Simon, F. Krammer, A serological assay to detect SARS-CoV-2 seroconversion in humans. Nat. Med. 26, 1033-1036 (2020). doi:10.1038/s41591-020-0913-5 Medline

15. D. J. Fitzgerald, P. Berger, C. Schaffitzel, K. Yamada, T. J. Richmond, I. Berger, Protein complex expression by using multigene baculoviral vectors. Nat. Methods 3, 1021-1032 (2006). doi:10.1038/nmeth983 Medline

16. To produce spike glycoprotein, we used here baculovirus-infected insect cells (Hi5) cultured in ESF921 media which contains cod liver oil as a nutrient supplement (Corey Jacklin, Expression Systems, personal communication). Cod liver oil comprises hundreds of FFAs including LA (38) which could be a possible source of the fatty acid. In parallel to our work, cryo-EM structures were determined of spike expressed in a mammalian system (HEK293) in serum and protein-free media $(39,40)$. The tube-shaped density we identified in our study is also present in those structures (but was not assigned or interpreted). We conclude that LA binding in the SARS-CoV-2 S pocket is thus not dependent on the expression system or media used.

17. D. Wrapp, N. Wang, K. S. Corbett, J. A. Goldsmith, C.-L. Hsieh, O. Abiona, B. S. Graham, J. S. McLellan, Cryo-EM structure of the 2019-nCoV spike in the prefusion conformation. Science 367, 1260-1263 (2020). doi:10.1126/science.abb2507 Medline

18. M. A. Tortorici, A. C. Walls, Y. Lang, C. Wang, Z. Li, D. Koerhuis, G.-J. Boons, B.-J. Bosch, F. A. Rey, R. J. de Groot, D. Veesler, Structural basis for human coronavirus attachment to sialic acid receptors. Nat. Struct. Mol. Biol. 26, 481-489 (2019). doi:10.1038/s41594-019-0233-y Medline

19. Y. Yuan, D. Cao, Y. Zhang, J. Ma, J. Qi, Q. Wang, G. Lu, Y. Wu, J. Yan, Y. Shi, X. Zhang, G. F. Gao, Cryo-EM structures of MERS-CoV and SARS-CoV spike glycoproteins reveal the dynamic receptor binding domains. Nat. Commun. 8, 15092 (2017). doi:10.1038/ncomms15092 Medline

20. J. M. Gullett, M. G. Cuypers, M. W. Frank, S. W. White, C. O. Rock, A fatty acidbinding protein of Streptococcus pneumoniae facilitates the acquisition of host polyunsaturated fatty acids. J. Biol. Chem. 294, 16416-16428 (2019). doi:10.1074/jbc.RA119.010659 Medline

21. J. Wang, E. J. Murphy, J. C. Nix, D. N. M. Jones, Aedes aegypti Odorant Binding Protein 22 selectively binds fatty acids through a conformational change in its $\mathrm{C}$ terminal tail. Sci. Rep. 10, 3300 (2020). doi:10.1038/s41598-020-60242-9 Medline

22. J. Shang, G. Ye, K. Shi, Y. Wan, C. Luo, H. Aihara, Q. Geng, A. Auerbach, F. Li, Structural basis of receptor recognition by SARS-CoV-2. Nature 581, 221-224 (2020). doi:10.1038/s41586-020-2179-y Medline

23. J. F. Glatz, J. H. Veerkamp, Removal of fatty acids from serum albumin by Lipidex 1000 chromatography. J. Biochem. Biophys. Methods 8, 57-61 (1983). doi:10.1016/0165-022X(83)90021-0 Medline

24. H. J. C. Berendsen, D. van der Spoel, R. van Drunen, GROMACS: A messagepassing parallel molecular dynamics implementation. Comput. Phys. Commun. 91, 43-56 (1995). doi:10.1016/0010-4655(95)00042-E

25. J. H. Beigel, K. M. Tomashek, L. E. Dodd, A. K. Mehta, B. S. Zingman, A. C. Kalil, E. Hohmann, H. Y. Chu, A. Luetkemeyer, S. Kline, D. Lopez de Castilla, R. W. Finberg, K. Dierberg, V. Tapson, L. Hsieh, T. F. Patterson, R. Paredes, D. A. Sweeney, W. R. Short, G. Touloumi, D. C. Lye, N. Ohmagari, M. Oh, G. M. Ruiz-Palacios, T. Benfield, G. Fätkenheuer, M. G. Kortepeter, R. L. Atmar, C. B. Creech, J. Lundgren, A. G. Babiker, S. Pett, J. D. Neaton, T. H. Burgess, T. Bonnett, M. Green, M. Makowski, A. Osinusi, S. Nayak, H. C. Lane, Remdesivir for the Treatment of Covid-19 Preliminary Report. N. Engl. J. Med. 10.1056/NEJMoa2007764 (2020). doi:10.1056/NEJMoa2007764

26. X. Ou, H. Guan, B. Qin, Z. Mu, J. A. Wojdyla, M. Wang, S. R. Dominguez, Z. Qian, S. Cui, Crystal structure of the receptor binding domain of the spike glycoprotein of human betacoronavirus HKU1. Nat. Commun. 8, 15216 (2017). doi:10.1038/ncomms 15216 Medline

27. Z. Li, A. C. A. Tomlinson, A. H. M. Wong, D. Zhou, M. Desforges, P. J. Talbot, S. Benlekbir, J. L. Rubinstein, J. M. Rini, The human coronavirus HCoV-229E Sprotein structure and receptor binding. eLife 8, e51230 (2019). doi:10.7554/eLife. 51230 Medline

28. A. C. Walls, M. A. Tortorici, B. Frenz, J. Snijder, W. Li, F. A. Rey, F. DiMaio, B.-J. Bosch, D. Veesler, Glycan shield and epitope masking of a coronavirus spike protein observed by cryo-electron microscopy. Nat. Struct. Mol. Biol. 23, 899905 (2016). doi:10.1038/nsmb.3293 Medline

29. J. Badger et al., Structural analysis of a series of antiviral agents complexed with human rhinovirus 14. Proc. Natl. Acad. Sci. U.S.A. 85, 3304-3308 (1988). doi:10.1073/pnas.85.10.3304 Medline

30. M. A. Oliveira, R. Zhao, W.-M. Lee, M. J. Kremer, I. Minor, R. R. Rueckert, G. D. Diana, D. C. Pevear, F. J. Dutko, M. A. McKinlay, M. G. Rossmann, The structure of human rhinovirus 16. Structure 1, 51-68 (1993). doi:10.1016/09692126(93)90008-5 Medline

31. V. Casanova, F. H. Sousa, C. Stevens, P. G. Barlow, Antiviral therapeutic approaches for human rhinovirus infections. Future Virol. 13, 505-518 (2018). doi:10.2217/fvl-2018-0016 Medline

32. K. K. W. To, C. C. Y. Yip, K. Y. Yuen, Rhinovirus - From bench to bedside. J. Formos. Med. Assoc. 116, 496-504 (2017). doi:10.1016/j.jfma.2017.04.009 Medline

33. B. Shen, X. Yi, Y. Sun, X. Bi, J. Du, C. Zhang, S. Quan, F. Zhang, R. Sun, L. Qian, W. Ge, W. Liu, S. Liang, H. Chen, Y. Zhang, J. Li, J. Xu, Z. He, B. Chen, J. Wang, H. Yan, Y. Zheng, D. Wang, J. Zhu, Z. Kong, Z. Kang, X. Liang, X. Ding, G. Ruan, N. Xiang, X. Cai, H. Gao, L. Li, S. Li, Q. Xiao, T. Lu, Y. Zhu, H. Liu, H. Chen, T. Guo, Proteomic and Metabolomic Characterization of COVID-19 Patient Sera. Cell 182, 59-72.e15 (2020). doi:10.1016/i.cell.2020.05.032 Medline 
34. C. M. Goodwin, S. Xu, J. Munger, Stealing the Keys to the Kitchen: Viral Manipulation of the Host Cell Metabolic Network. Trends Microbiol. 23, 789-798 (2015). doi:10.1016/i.tim.2015.08.007 Medline

35. J. W. Schoggins, G. Randall, Lipids in innate antiviral defense. Cell Host Microbe 14 , 379-385 (2013). doi:10.1016/i.chom.2013.09.010 Medline

36. M. M. Zaman, C. R. Martin, C. Andersson, A. Q. Bhutta, J. E. Cluette-Brown, M. Laposata, S. D. Freedman, Linoleic acid supplementation results in increased arachidonic acid and eicosanoid production in CF airway cells and in cftr-/. transgenic mice. Am. J. Physiol. Lung Cell. Mol. Physiol. 299, L599-L606 (2010). doi:10.1152/ajplung.00346.2009 Medline

37. I. Kimura, A. Ichimura, R. Ohue-Kitano, M. Igarashi, Free Fatty Acid Receptors in Health and Disease. Physiol. Rev. 100, 171-210 (2020). doi:10.1152/physrev.00041.2018 Medline

38. S. Hauff, W. Vetter, Quantitation of cis- and trans-monounsaturated fatty acids in dairy products and cod liver oil by mass spectrometry in the selected ion monitoring mode. J. Agric. Food Chem. 57, 3423-3430 (2009). doi:10.1021/if803665u Medline

39. Y. Cai, J. Zhang, T. Xiao, H. Peng, S. M. Sterling, R. M. Walsh Jr., S. Rawson, S. RitsVolloch, B. Chen, Distinct conformational states of SARS-CoV-2 spike protein. Science eabd4251 (2020). doi:10.1126/science.abd4251 Medline

40. A. G. Wrobel, D. J. Benton, P. Xu, C. Roustan, S. R. Martin, P. B. Rosenthal, J. J. Skehel, S. J. Gamblin, SARS-CoV-2 and bat RaTG13 spike glycoprotein structures inform on virus evolution and furin-cleavage effects. Nat. Struct. Mol. Biol. 27, 763-767 (2020). doi:10.1038/s41594-020-0468-7 Medline

41. I. Berger, D. J. Fitzgerald, T. J. Richmond, Baculovirus expression system for heterologous multiprotein complexes. Nat. Biotechnol. 22, 1583-1587 (2004). doi:10.1038/nbt1036 Medline

42. M. M. Vork, J. F. Glatz, D. A. Surtel, G. J. van der Vusse, Assay of the binding of fatty acids by proteins: Evaluation of the Lipidex 1000 procedure. Mol. Cell. Biochem. 98, 111-117 (1990). doi:10.1007/BF00231374 Medline

43. Q. Wang, S. Rizk, C. Bernard, M. P. Lai, D. Kam, J. Storch, R. E. Stark, Protocols and pitfalls in obtaining fatty acid-binding proteins for biophysical studies of ligandprotein and protein-protein interactions. Biochem. Biophys. Rep. 10, 318-324 (2017). doi:10.1016/j.bbrep.2017.05.001 Medline

44. M. Fairhead, M. Howarth, Site-specific biotinylation of purified proteins using BirA. Methods Mol. Biol. 1266, 171-184 (2015). doi:10.1007/978-1-4939-2272-7.12 Medline

45. S. Q. Zheng, E. Palovcak, J.-P. Armache, K. A. Verba, Y. Cheng, D. A. Agard, MotionCor2: Anisotropic correction of beam-induced motion for improved cryoelectron microscopy. Nat. Methods 14, 331-332 (2017). doi:10.1038/nmeth.4193 Medline

46. A. Rohou, N. Grigorieff, CTFFIND4: Fast and accurate defocus estimation from electron micrographs. J. Struct. Biol. 192, 216-221 (2015). doi:10.1016/i.jsb.2015.08.008 Medline

47. S. H. Scheres, RELION: Implementation of a Bayesian approach to cryo-EM structure determination. J. Struct. Biol. 180, 519-530 (2012). doi:10.1016/i.jsb.2012.09.006 Medline

48. T. D. Goddard, C. C. Huang, T. E. Ferrin, Visualizing density maps with UCSF Chimera. J. Struct. Biol. 157, 281-287 (2007). doi:10.1016/i.jsb.2006.06.010 Medline

49. P. Emsley, B. Lohkamp, W. G. Scott, K. Cowtan, Features and development of Coot. Acta Crystallogr. D 66, 486-501 (2010). doi:10.1107/S0907444910007493 Medline

50. T. C. Terwilliger, O. V. Sobolev, P. V. Afonine, P. D. Adams, Automated map sharpening by maximization of detail and connectivity. Acta Crystallogr. D 74, 545-559 (2018). doi:10.1107/S2059798318004655 Medline

51. N. W. Moriarty, R. W. Grosse-Kunstleve, P. D. Adams, electronic Ligand Builder and Optimization Workbench (eLBOW): A tool for ligand coordinate and restraint generation. Acta Crystallogr. D 65, 1074-1080 (2009). doi:10.1107/S0907444909029436 Medline

52. D. Liebschner, P. V. Afonine, M. L. Baker, G. Bunkóczi, V. B. Chen, T. I. Croll, B. Hintze, L.-W. Hung, S. Jain, A. J. McCoy, N. W. Moriarty, R. D. Oeffner, B. K. Poon, M. G. Prisant, R. J. Read, J. S. Richardson, D. C. Richardson, M. D. Sammito, O. V. Sobolev, D. H. Stockwell, T. C. Terwilliger, A. G. Urzhumtsev, L. L. Videau, C. J. Williams, P. D. Adams, Macromolecular structure determination using X-rays, neutrons and electrons: Recent developments in Phenix. Acta Crystallogr. D 75, 861-877 (2019). doi:10.1107/S2059798319011471 Medline

53. V. B. Chen, W. B. Arendall 3rd, J. J. Headd, D. A. Keedy, R. M. Immormino, G. J. Kapral, L. W. Murray, J. S. Richardson, D. C. Richardson, MolProbity: All-atom structure validation for macromolecular crystallography. Acta Crystallogr. D 66 , 12-21 (2010). doi:10.1107/S0907444909042073 Medline

54. B. A. Barad, N. Echols, R. Y.-R. Wang, Y. Cheng, F. DiMaio, P. D. Adams, J. S. Fraser, EMRinger: Side chain-directed model and map validation for 3D cryo-electron microscopy. Nat. Methods 12, 943-946 (2015). doi:10.1038/nmeth.3541 Medline 55. J. L. Daly, B. Simonetti, C. Antón-Plágaro, M. Kavanagh Williamson, D. K. Shoemark, L. Simón-Gracia, K. Klein, M. Bauer, R. Hollandi, U. F. Greber, P. Horvath, R. B. Sessions, A. Helenius, J. A. Hiscox, T. Teesalu, D. A. Matthews, A. D. Davidson, P. J. Cullen, Y. Yamauchi, Neuropilin-1 is a host factor for SARS-CoV-2 infection. bioRxiv (2020). https://doi.org/10.1101/2020.06.05.134114.

56. A. D. Davidson, M. K. Williamson, S. Lewis, D. Shoemark, M. W. Carroll, K. J. Heesom, M. Zambon, J. Ellis, P. A. Lewis, J. A. Hiscox, D. A. Matthews, Characterisation of the transcriptome and proteome of SARS-CoV-2 reveals a cell passage induced in-frame deletion of the furin-like cleavage site from the spike glycoprotein. Genome Med. 12, 68 (2020). doi:10.1186/s13073-020-00763-0 Medline

57. V. M. Corman, O. Landt, M. Kaiser, R. Molenkamp, A. Meijer, D. K. W. Chu, T. Bleicker, S. Brünink, J. Schneider, M. L. Schmidt, D. G. J. C. Mulders, B. L. Haagmans, B. van der Veer, S. van den Brink, L. Wijsman, G. Goderski, J.-L. Romette, J. Ellis, M. Zambon, M. Peiris, H. Goossens, C. Reusken, M. P. G. Koopmans, C. Drosten, Detection of 2019 novel coronavirus (2019-nCoV) by realtime RT-PCR. Euro Surveill. 25, 2000045 (2020). doi:10.2807/15607917 ES 20202532000045 Medline

58. R. A. Laskowski, M. W. MacArthur, D. S. Moss, J. M. P. Thornton, PROCHECK - a program to check the stereochemical quality of protein structures. J. Appl. Cryst. 26, 283-291 (1993). doi:10.1107/S0021889892009944

59. A. W. Sousa da Silva, W. F. Vranken, ACPYPE - AnteChamber PYthon Parser interfacE. BMC Res. Notes 5, 367 (2012). doi:10.1186/1756-0500-5-367 Medline

60. J. Wang, R. M. Wolf, J. W. Caldwell, P. A. Kollman, D. A. Case, Development and testing of a general amber force field. J. Comput. Chem. 25, 1157-1174 (2004). doi:10.1002/icc.20035 Medline

61. D. A. Case, T. E. Cheatham 3rd, T. Darden, H. Gohlke, R. Luo, K. M. Merz Jr., A. Onufriev, C. Simmerling, B. Wang, R. J. Woods, The Amber biomolecular simulation programs. J. Comput. Chem. 26, 1668-1688 (2005) doi:10.1002/jcc.20290 Medline

62. K. Lindorff-Larsen, S. Piana, K. Palmo, P. Maragakis, J. L. Klepeis, R. O. Dror, D. E. Shaw, Improved side-chain torsion potentials for the Amber ff99SB protein force field. Proteins 78, 1950-1958 (2010). doi:10.1002/prot.22711 Medline

63. W. Humphrey, A. Dalke, K. Schulten, VMD: Visual molecular dynamics. J. Mol. Graph. 14, 33-38 (1996). doi:10.1016/0263-7855(96)00018-5 Medline

\section{ACKNOWLEDGMENTS}

We thank all members of the Berger and Schaffitzel teams as well as Gunjita Singh, Yohei Yamauchi and David Matthews (University of Bristol, UK) for their assistance. We thank Florian Krammer (Icahn School of Medicine, USA) for kindly sharing expression plasmids. We thank Veronica Chang and Radu Aricescu (MRC-LMB, UK) for kind gift of S expressed in HEK293. We are indebted to Adam Finn (Bristol UNCOVER Group and Children's Vaccine Centre, Bristol Medical School), Jeremy Tavaré (School of Biochemistry, Bristol), Kathleen Gillespie (Diabetes and Metabolism Unit, Southmead Hospital, Univ. of Bristol) and Donald Fitzgerald MD (Quest Imaging Medical Associates, USA) for helpful discussions and careful reading of the manuscript. We thank Simon Burbidge, Thomas Batstone and Matt Williams for computation infrastructure support. We would like to thank the Advanced Computing Research Centre (ACRC) at the University of Bristol for access to BlueCryo, BlueCrystal Phase 4 and BlueGEM, and the UK HECBioSim for access to the UK supercomputer, ARCHER. We are particularly grateful to Thiru Thangarajah (Genscript) for early access to Genscript's cPass SARS-CoV-2 Neutralization Antibody Detection/Surrogate Virus Neutralization Test Kit (L00847). We thank Sebastian Fabritz and the Core Facility for Mass Spectrometry at the Max Planck Institute for Medical Research for their support on MS measurements. Funding: This research received support 
from the Elizabeth Blackwell Institute for Health Research and the EPSRC Impact Acceleration Account EP/R511663/1, University of Bristol, from BrisSynBio a BBSRC/EPSRC Research Centre for synthetic biology at the University of Bristol (BB/L01386X/1) (to I.B., A.J.M., D.K.S.) and from the BBSRC (BB/P000940/1) (to C.S. and I.B.). This work received generous support from the Oracle Higher Education and Research program to enable cryo-EM data processing using Oracle's high-performance public cloud infrastructure (https://cloud.oracle.com/en US/cloud-infrastructure) and the EPSRC through a COVID-19 project award via HECBioSim to access ARCHER (A.J.M.). We acknowledge support and assistance by the Wolfson Bioimaging Facility and the GW4 Facility for High-Resolution Electron Cryo-Microscopy funded by the Wellcome Trust (202904/Z/16/Z and 206181/Z/17/Z) and BBSRC (BB/R000484/1). The authors would like to acknowledge support of the University of Bristol's Alumni and Friends, which funded the ImageXpress Pico Imaging System. O.S. acknowledges support from the Elisabeth Muerer Foundation, the Max Planck School Matter to Life and the Heidelberg Biosciences International Graduate School. A.D.D. is supported by the United States Food and Drug Administration (HHSF223201510104C) and UK Research and Innovation/Medical Research Council (MRC) (MR/V027506/1). M.K.W is supported by MRC grants MR/R020566/1 and MR/V027506/1 (awarded to A.D.D). A.J.M. is supported the British Society for Antimicrobial Chemotherapy (BSAC-COVID-30) and the EPSRC (EP/M022609/1, CCP-BioSim). I.B. acknowledges support from the EPSRC Future Vaccine Manufacturing and Research Hub (EP/R013764/1). C.S. and I.B. are Investigators of the Wellcome Trust (210701/Z/18/Z; 106115/Z/14/Z). Author contributions: C.S. and I.B. conceived and guided the study. F.G., K.G. and J.C. produced, purified and analyzed sample, K.G. carried out biochemical experiments, S.K.N.Y. and U.B. prepared grids and collected EM data, S.K.N.Y., U.B., K.G and C.T. carried out image analysis and model building. A.D.D., M.K.W. and R.M. performed all live virus $\mathrm{CL} 3$ work and analyzed data. D.K.S. and A.J.M. performed all MD simulations. O.S. and J.S. performed and interpreted mass spectrometry. C.T., K.G., D.F., I.B. and C.S. interpreted results. D.F., I.B. and C.S. wrote the manuscript with input from all authors. Competing interests: The authors declare competing interests. I.B and F.G. report shareholding in Imophoron Ltd. unrelated to this Correspondence. I.B. and D.F. report shareholding in Geneva Biotech Sàrl related to this Correspondence. Patent applications describing methods and material compositions based on the present observations have been filed. Data and materials availability: Datasets generated during the current study have been deposited in the Electron Microscopy Data Bank (EMDB) under accession numbers EMD-11145 (C3 closed conformation), EMD11144 (C1 closed conformation) and EMD-11146 (open conformation), and in the Protein Data Bank (PDB) under accession numbers: 6ZB5 (C3 closed conformation) and 6ZB4 (C1 closed conformation). Reagents are available from I.B. and C.S. under a material transfer agreement with the University of Bristol. This work is licensed under a Creative Commons Attribution 4.0 International (CC BY 4.0) license, which permits unrestricted use, distribution, and reproduction in any medium, provided the original work is properly cited. To view a copy of this license, visit https://creativecommons.org/licenses/by/4.0/. This license does not apply to figures/photos/artwork or other content included in the article that is credited to a third party; obtain authorization from the rights holder before using such material.

\section{SUPPLEMENTARY MATERIALS}

\section{science.sciencemag.org/cgi/content/full/science.abd3255/DC1}

Materials and Methods

Figs. S1 to S12

Tables S1 to S3

References (41-63)

MDAR Reproducibility Checklist

Movies S1 to S4

15 June 2020; accepted 16 September 2020

Published online 21 September 2020

10.1126/science.abd3255 

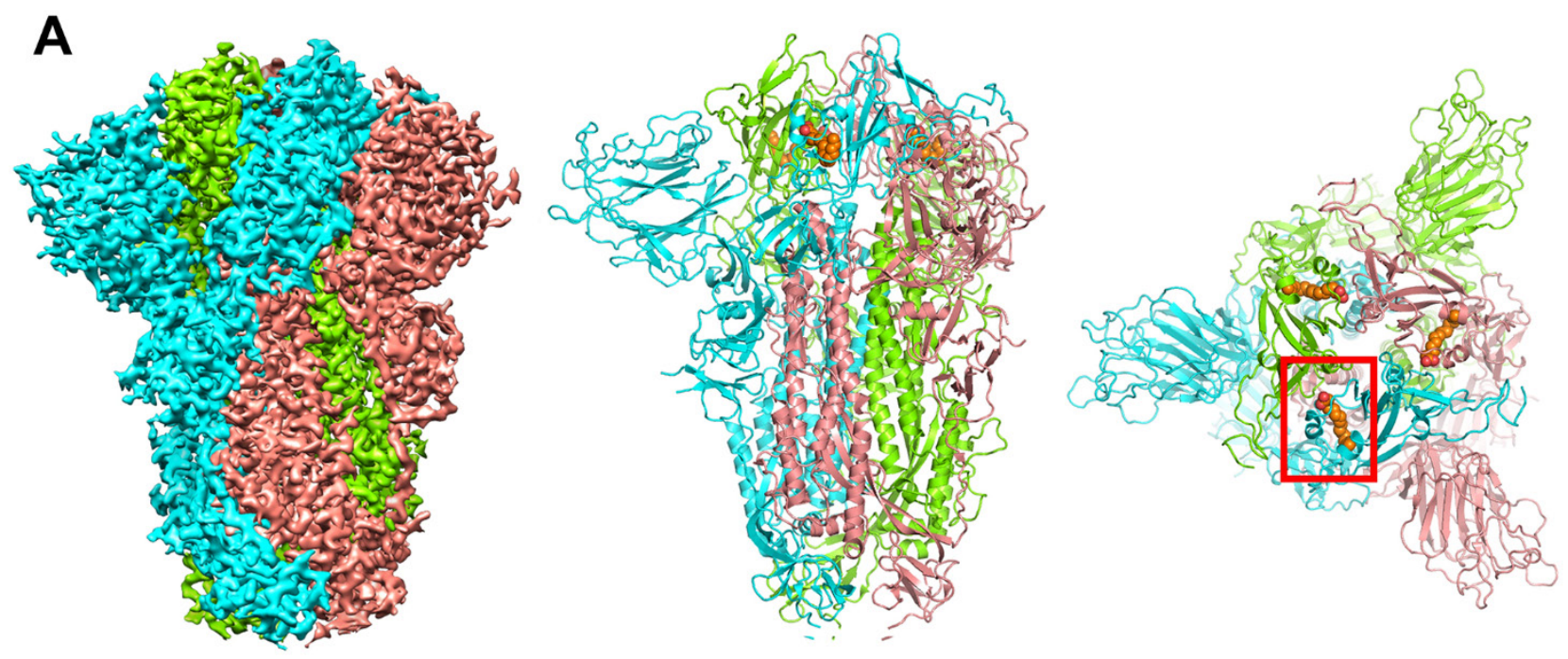

B

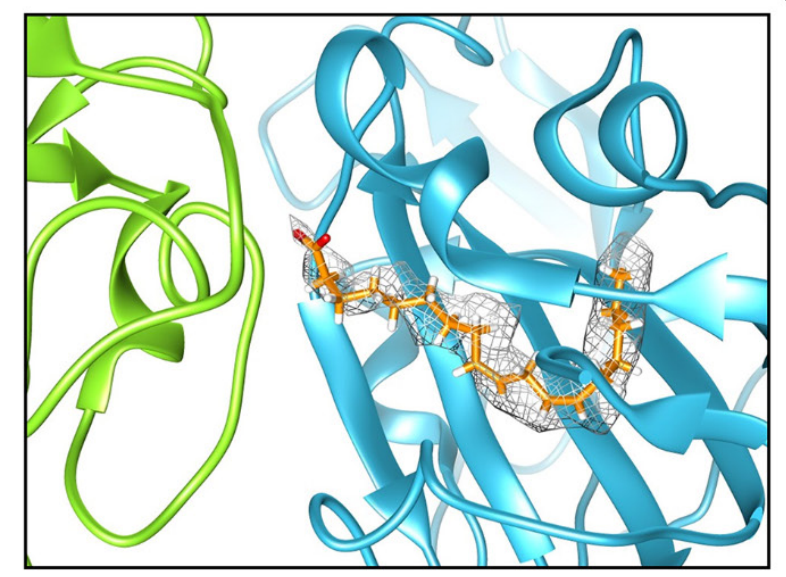

D

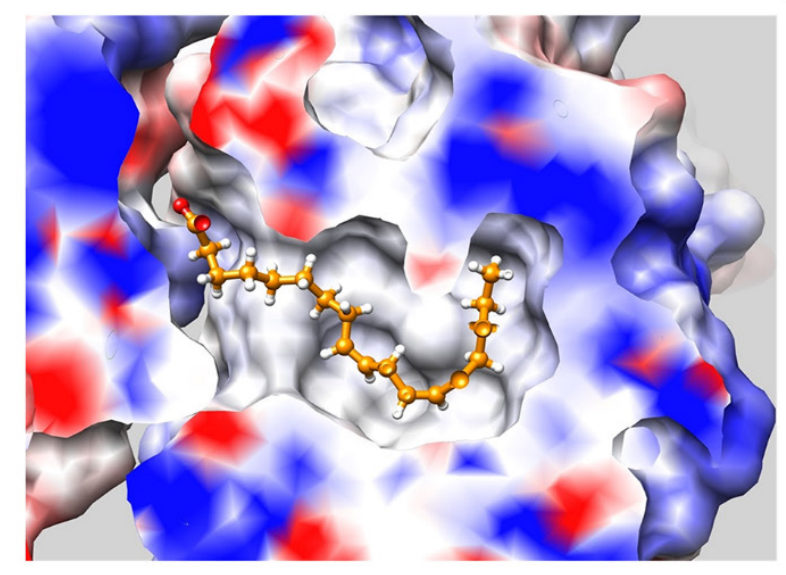

C

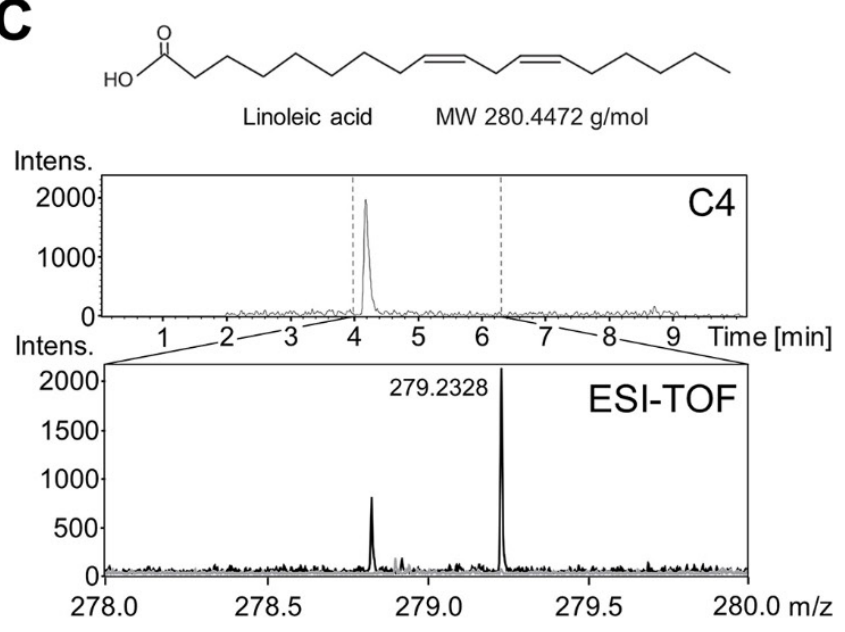

E

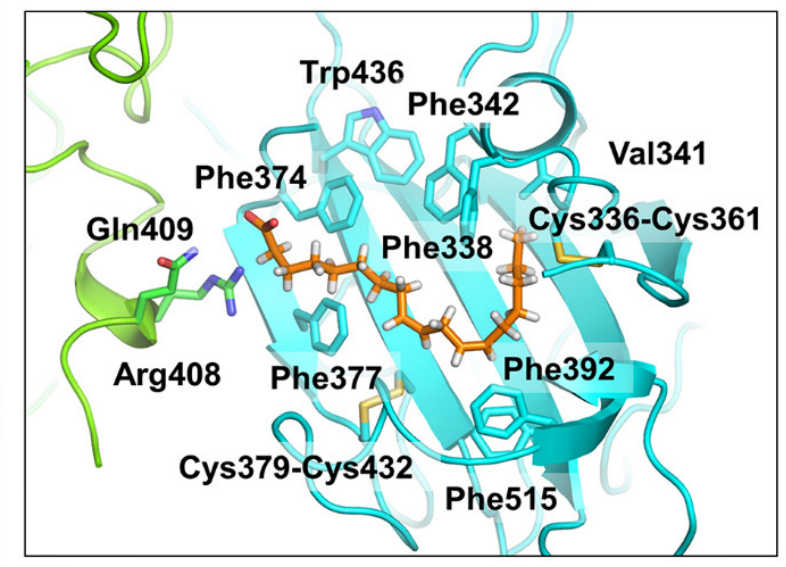


Fig. 1. Cryo-EM structure of SARS-CoV-2 spike linoleic acid complex. (A) Cryo-EM density of spike trimer (left). Monomers in cyan, green and pink, respectively. The structure in a cartoon representation in a front (middle) and top view (right). Bound LA illustrated as spheres colored in orange. One LAbinding pocket is boxed in red. (B) Composite LA-binding pocket formed by adjacent RBDs. Tubeshaped EM density is shown. (C) LC-MS of purified S. Chemical structure and molecular weight of LA (top), C4 column elution profile (middle) and ESI-TOF of wash solution (gray) and C4 peak elution fraction (black) with peak molecular weight indicated (bottom). (D) Hydrophobic LA-binding pocket in a surface representation illustrating excellent fit of bound LA (orange, sticks and balls representation). Blue and red indicate positive and negative surface charge, respectively. (E) LA interactions with amino acids in the binding pocket. The acidic LA headgroup is in the vicinity of an arginine (408) and a glutamine (409). 


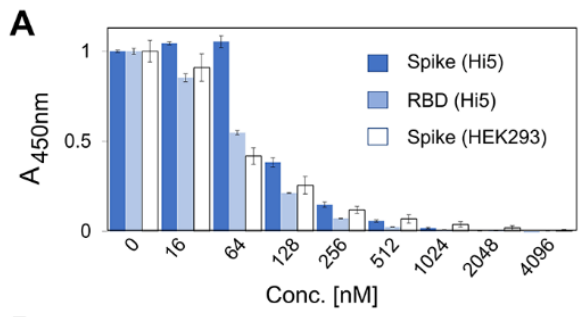

B

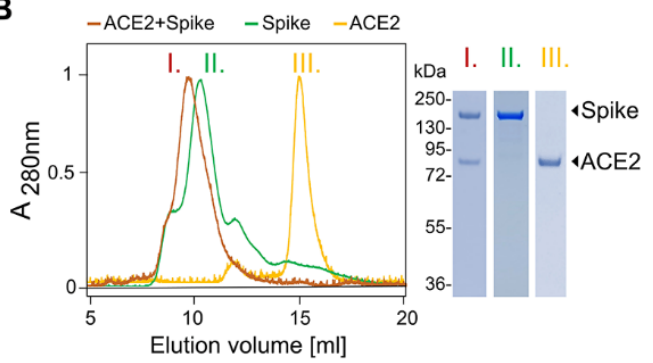

C

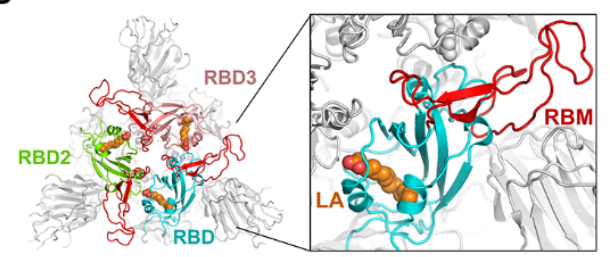

D
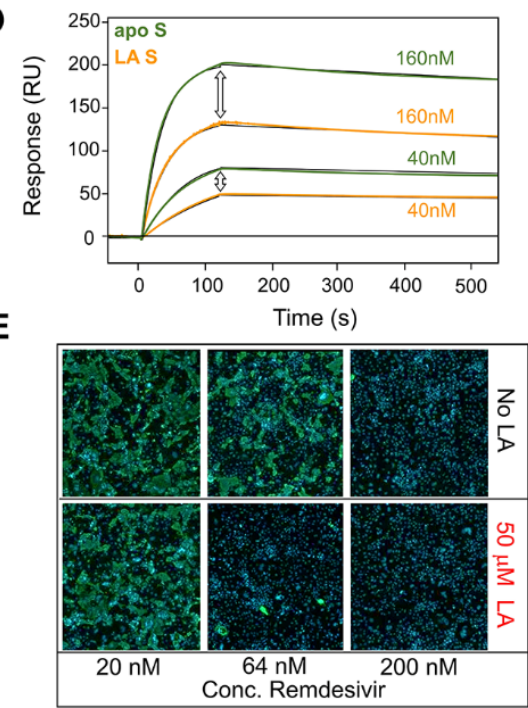

$\mathbf{F}$

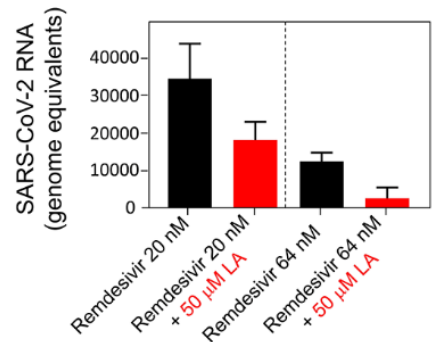

Fig. 2. Functional characterization of LA-bound SARS-CoV-2 spike. (A) Insect cell ( $\mathrm{Hi5}$ ) expressed spike (dark blue bars), insect cell expressed RBD (light blue bars) and mammalian (HEK293) expressed spike (white bars) in competition ELISAs utilizing immobilized ACE2. Error bars: standard deviations, three replicates. (B) LA-bound SARS-CoV-2 spike protein interaction with ACE2 was analyzed by size exclusion chromatography evidencing complex formation. SEC profiles (left) for ACE2 (yellow, III), LAbound spike (green, II) and a mixture of ACE2 and LA-bound spike (orange, I) are shown. Peak fractions (I.-III.) were analyzed by SDS-PAGE evidencing the expected proteins (right). (C) LA-bound S glycoprotein trimer top view with RBDs shown in cyan, green and pink respectively (left). In each RBD subunit the motif responsible for ACE2 binding (RBM) is in red, and LA is shown as spheres colored in orange. A close-up view into the cyan RBD (right) shows that the RBM is fully ordered, and LA and RBM are not in direct contact. (D) SPR analysis of the binding of LA-bound spike trimer (orange curves) and apo spike trimer (green curves) to immobilized ACE2. Apo spike and LA-bound spike were diluted to concentrations of $40 \mathrm{nM}$ and 160 $\mathrm{nM}$, respectively. Black lines correspond to a global fit using a 1:1 binding model. (E) Synergistic effect of LA and remdesivir on SARS-CoV-2 viral replication. Effects of varying doses of remdesivir $\pm 50 \mu \mathrm{M} L A$ on virus infection are shown. Human Caco-2 ACE2+ cells were infected with SARSCoV-2 and then treated with varying doses of remdesivir $\pm 50 \mu \mathrm{M}$ LA. At 96 hours post-infection, cells were fixed and infected cells detected by immunofluorescence assay using an anti- $\mathrm{N}$ antibody (green). Cell nuclei were stained by DAPI (blue). Representative images corresponding to the remdesivir dose range $20-200 \mathrm{nM}$ are shown. (F) The amount of extracellular virus present in wells $(n=3)$ at the dose combinations shown was determined by qRT-PCR. 


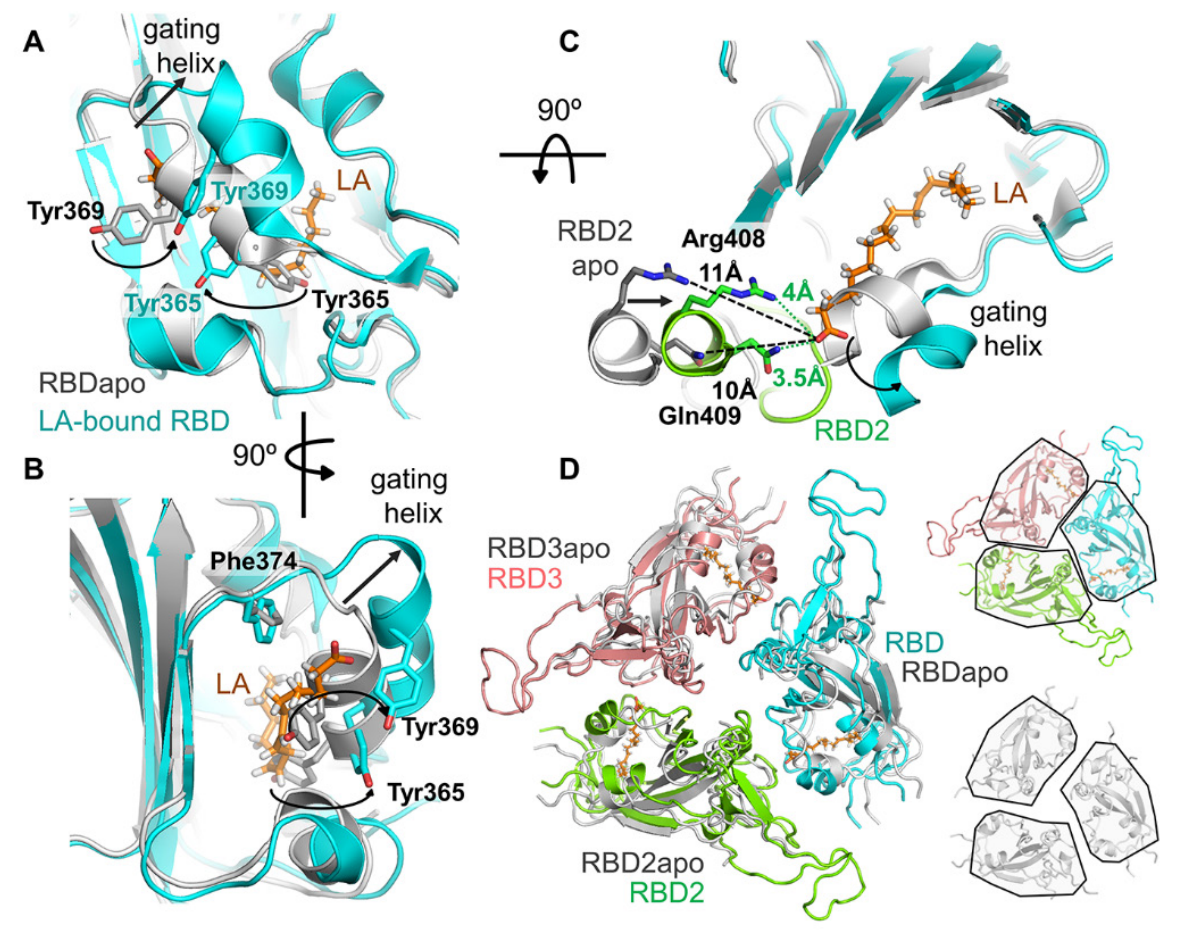

Fig. 3. Comparison of LA-bound and apo $S$ structures. (A) Superimposition of LA-bound SARS-CoV-2 (cyan RBD) and the ligandfree "apo" RBD (gray) (PDBID 6VXX (7)). The gating helix at the entrance of the hydrophobic pocket moves by $6 \AA$ in the presence of LA. Tyr365 and Tyr369 swing away avoiding clashes with LA (orange). Black arrows indicate the rearrangements. (B) The same structure as panel A rotated by $90^{\circ}$ as indicated, showing the entrance of the hydrophobic pocket. (C) Formation of a composite LA-binding pocket by two adjacent RBDs in LA-bound $S$ involves a $\sim 5 \AA$ movement of RBD2 (green) toward RBD1 (cyan) compared to apo S (gray). (D) Superimposition of the RBD trimer of apo $S$ (gray) and LA-bound $S$ (RBD1 cyan, RBD2 green, RBD3 pink, LA orange) is shown (left). The individual RBD trimers are depicted for LA-bound S (right, top) and apo $S$ (right, bottom) with RBDs boxed in black, highlighting the compaction of RBDs in the LA-bound S structure. 
A

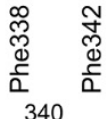

350

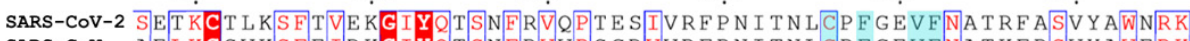
SARS-COV AELKCSVKSFEIDKGIYOTSNFRVVP S GDVVRFPNITNLCPFGEVFNATKFPSVYAWERK MERS-COV SQLHCSYESFDVESGVYSVISEEAKP SGSVVEQAEG. VECDFSPLLSG. TPPQVYNFKRI hCoV-OC43 SE I KCKT OS I AP P T GVYELNG Y TVQP I ADVYRRKP D LP N CN I E AW LNDKS VP SP LNWERK

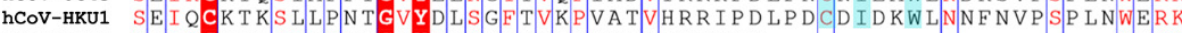
hCoV-229E NRLRCDQLSEDVPDGEYSTSP I QSVELPVSIVSLPV ............... YHKH hCov-NL63 EKLQCEH LQEG L QDGFYSANF LD DNVLPETYVALP I . . . . . . . . . . . . YY
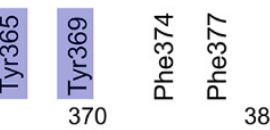

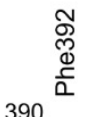

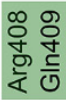

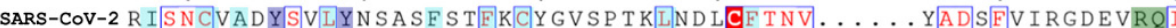
SARS-COV KISNCVADYSVIYYNSTFF STFKCYGVSATKLNDLCF SNV $\ldots \ldots . .$. YADSFVVKGDDVRQ

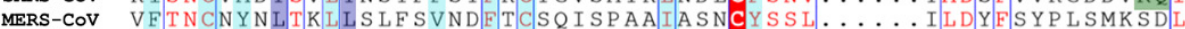
COV hCov-C43 hCoV-HU1 ION

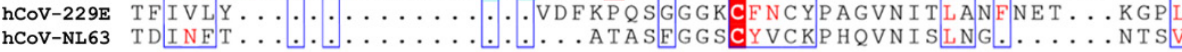

B

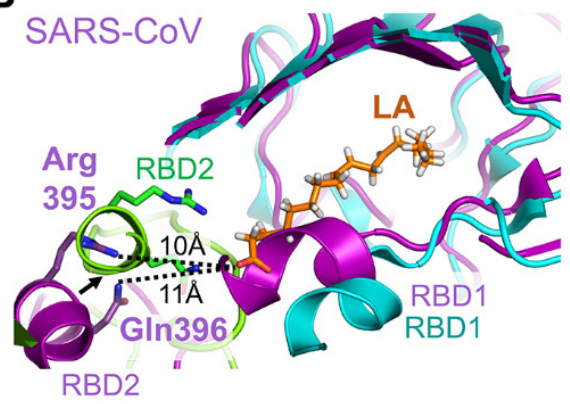

C
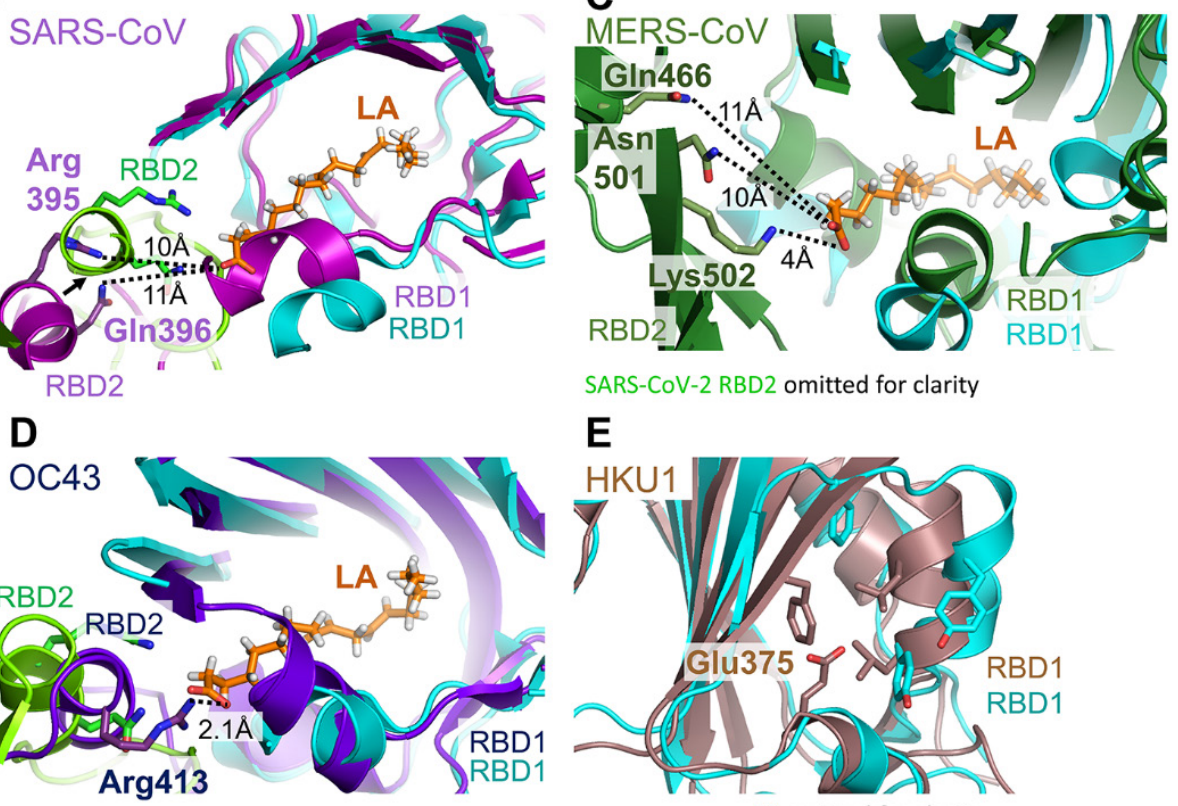

SARS-CoV-2 RBD2 omitted for clarity

E

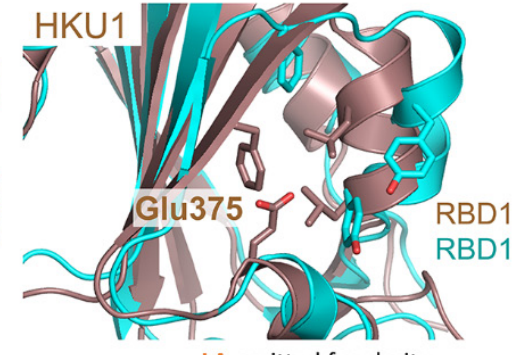

LA omitted for clarity

Fig. 4. Human coronavirus RBD architectures. (A) Alignments of the seven CoV strains that can infect humans, highlighting conserved residues. Residues lining the hydrophobic pocket are underlaid (cyan). Gating helix residues are marked (purple). Residues positioned to interact with the LA polar headgroup are underlaid in green. Glu375 in HKU1 is underlaid in red (cf. panel E). (B) Superimposition of RBD1 of LA-bound SARS-CoV-2 (RBD2 in green) with RBD1 of ligand free "apo" SARS-CoV (RBD1 and RBD2 magenta, PDBID 5X58 (19)) indicates a conservation of the composite binding pocket. (C) Superimposition of RBD1 of LA-bound SARS-CoV-2 (RBD2 is omitted for clarity) with RBD1 of MERS-CoV (RBD1 and RBD2 forest green, PDBID 5X5F (19)). (D) Superimposition of RBD1 of LA-bound SARS-CoV-2 (RBD2 in green) with RBD1 of OC43 (RBD1 and RBD2 purple, PDBID 6NZK (18)). (E) Superimposition of LA-bound SARS-CoV-2 RBD with HKU1 RBD (brown, PDBID 5GNB (26)). LA is omitted in SARS-CoV-2 RBD for clarity. 